\title{
Effect of strain and temperature on the threshold displacement energy in body-centered cubic iron
}

\author{
Benjamin Beeler ${ }^{\mathrm{a}, \mathrm{b}, *}$, Mark Asta $^{\mathrm{b}}$, Peter Hosemann $^{\mathrm{c}}$, Niels Grønbech-Jensen $^{\mathrm{a}, \mathrm{d}}$ \\ ${ }^{a}$ Department of Mechanical and Aerospace Engineering, University of California, Davis, CA 95616 \\ ${ }^{b}$ Department of Materials Science, University of California, Berkeley, CA 94720 \\ ${ }^{c}$ Department of Nuclear Engineering, University of California, Berkeley, CA 94720 \\ ${ }^{d}$ Department of Mathematics, University of California, Davis, CA 95616
}

\begin{abstract}
The threshold displacement energy (TDE) is the minimum amount of kinetic energy required to displace an atom from its lattice site. The magnitude of the TDE displays significant variance as a function of the crystallographic direction, system temperature and applied strain, among a variety of other factors. It is critically important to determine an accurate value of the TDE in order to calculate the total number of displacements due to a given irradiation condition, and thus to understand the materials response to irradiation. In this study, molecular dynamics simulations have been performed to calculate the threshold displacement energy in body-centered cubic iron as a function of strain and temperature. With applied strain, a decrease of the TDE of up to approximately $14 \mathrm{eV}$ was observed. A temperature increase from 300 $\mathrm{K}$ to $500 \mathrm{~K}$ can result in an increase of the TDE of up to approximately $9 \mathrm{eV}$.
\end{abstract}

\section{Introduction}

Structural materials for nuclear fission and fusion applications experience a dynamic temperature, irradiation and mechanical loading environment. Understanding materials response to irradiation in this dynamic environment is one of the key issues for the design and operation of nuclear power systems. Lifetime and operational restrictions are typically influenced by limitations associated with the degradation of structural materials properties under irradiation. The ability to accurately predict and understand behavior of structural materials under short term and long term irradiation is thus important for designing improved materials for nuclear power applications.

Extensive computational research has been performed in order to better understand radiation damage in widely used structural materials, including ferritic alloys based on body-centered cubic (BCC) iron (Fe) 1, 2, 3, 4, 5, 6, 7. Common areas of interest with regards to radiation damage simulations have been general damage behavior [8, 9, 10, variations in primary knock-on atom (PKA) energy [11, 12, 13, variations in simulation temperature during irradiation [4, 9, 14, 15, 16, the behavior and effect of extrinsic particles

\footnotetext{
* Corresponding author

Email address: bwbeeler@ucdavis.edu (Benjamin Beeler)
} 
[17, 18, 19, 20, and the effect of strain [21, 22, 23. However, there have been fewer modeling investigations the total number of displacements due to a given irradiation condition, and thus understand the materials response to irradiation.

There is a long, but somewhat limited, history of investigating the TDE in BCC Fe. Nordlund, et al. [26] provide a comprehensive review of the early history of TDE studies and it will not be repeated here. A as a function of angle with a Finnis-Sinclair potential. Ackland, et al. 27] performed a similar study at 0 $\mathrm{K}$ using a many-body Finnis-Sinclair potential to investigate the effects of dilute $\mathrm{Cu}$ solute additions on the TDE. For application in engineering environments, the TDE needs to be studied at temperature and under non-zero strain conditions. Nordlund et al. [26] performed an extensive study of 11 separate interatomic potentials to investigate the lower bound of the TDE in BCC Fe at $36 \mathrm{~K}$. Zolnikov, et al. [28] studied the TDE in BCC iron and vanadium with high applied tetragonal shear and varying temperature, with an emphasis on radiation induced plastic deformation. Other studies have been performed to investigate the TDE in other material systems, such as nickel, rutile and diamond [29, 30, 16, 31]. A related study on the effect of strain on formation energy in Fe was studied via first principles by Chen et al. [32.

A recent study of the effects of applied strain on radiation damage generation in BCC Fe [21] showed that under certain strain conditions, there can be significant increases or decreases in the amount of defect accumulation from a given cascade. This led to a brief investigation into the displacement energy for a single PKA direction in a system with and without strain. It was shown that applied hydrostatic expansion leads to a decrease in the threshold displacement energy. A more expansive investigation into the TDE as a function of strain and temperature is thus warranted, and is the focus of this study.

In this paper, molecular dynamics (MD) [33, 34 simulations have been performed to calculate the TDE in BCC Fe at $300 \mathrm{~K}$ and $500 \mathrm{~K}$, under three separate strain conditions - unstrained, applied tetragonal shear (Bain strain), and applied hydrostatic expansion - for ten individual PKA directions. The probability of Frenkel pair production is determined as a function of PKA direction, strain and temperature. Probability curves are averaged to determine an effective value of the TDE, above which a Frenkel pair is more than 50 $\%$ likely to form. The minimum amount of kinetic energy required to create a Frenkel pair is also calculated as a function of strain, temperature and PKA direction. 


\section{Computational Details}

Molecular dynamics simulations are performed utilizing the LAMMPS [35] software package and the for the TDE needs to be utilized [26, 25]. This average value is obtained from generating probability curves (probability of generating a Frenkel pair as a function of PKA energy) for respective PKA directions. 
These probability curves can then be averaged over all directions to create an angle-integrated displacement

determined to be the median $\operatorname{TDE}\left(\mathrm{E}_{\mathrm{d} \text {,med }}^{\mathrm{pp}}\right)$, where pp stands for production probability. This value takes into account not only displacement of atoms, but allows for subsequent recombination in the time-frame of the 6 ps simulation following the PKA event. Both the $\mathrm{E}_{\mathrm{d} \text {,med }}^{\mathrm{pp}}$ and the $\mathrm{E}_{\mathrm{d}}^{\mathrm{l}}$ are calculated in this work.

\section{Results}

\subsection{Median Threshold Displacement Energy}

In this section, $\mathrm{E}_{\mathrm{d} \text {,med }}^{\mathrm{pp}}$ is determined through a variety of simulations. The results in Figure 1 outline the probability of Frenkel pair production as a function of the PKA energy for each of the ten random directions investigated. Each graph includes data points and fourth-order polynomial fits for an unstrained system, a system with applied $2 \%$ hydrostatic expansion and a system with applied $5 \%$ Bain strain. In these graphs, the red square data points and the red dashed line denote a system without external applied strain, the blue diamond data points and the blue solid line denote a system with $2 \%$ hydrostatic expansion. and the green triangle data points and the green dashed line denote a system with $5 \%$ Bain strain.

There are numerous interesting details from these ten graphs. The first is the general behavior of the probability of Frenkel pair production as a function of PKA energy. In accordance with previous results [26], there are large ranges in energy for nearly all investigated directions where the probability to create a Frenkel pair is finite and different from one. This suggests it would be more accurate to utilize the entire probability versus PKA energy curve to determine the actual displacements per atom under irradiation. Also, there often exists a plateauing of the probability curve. This can be understood as due to excess heat leading to increased recombination. As PKA energies increase, there is locally an increase in the temperature, this can more defects being produced. These competing effects yield this plateauing behavior. As the PKA energy increases, it finally becomes large enough to overcome this increase in recombination, leading to an increase in the probability of defect formation.

The second key feature of the graphs is the relative probabilities for a given PKA energy with and without applied strain. Overall, there is a clear trend that the application of $2 \%$ hydrostatic tensile strain shifts the probability curves to lower energies, suggesting that the TDE is lower under tensile strains. This point will be discussed in further detail below. There is some statistical variation in the data, but there exists an overall trend of increasing probability for Frenkel pair creation in the systems with applied tensile strain. This shows that the TDE is in fact affected by applied strain. For comparisons between unstrained system and systems with applied Bain strain, the trend is less clear. However, in most cases application of Bain strain results in a shift left of the probability curve, and thus a decrease in the TDE. Thirdly, it is clear that the general behavior of the probability versus energy curves varies greatly as a function of PKA direction. 


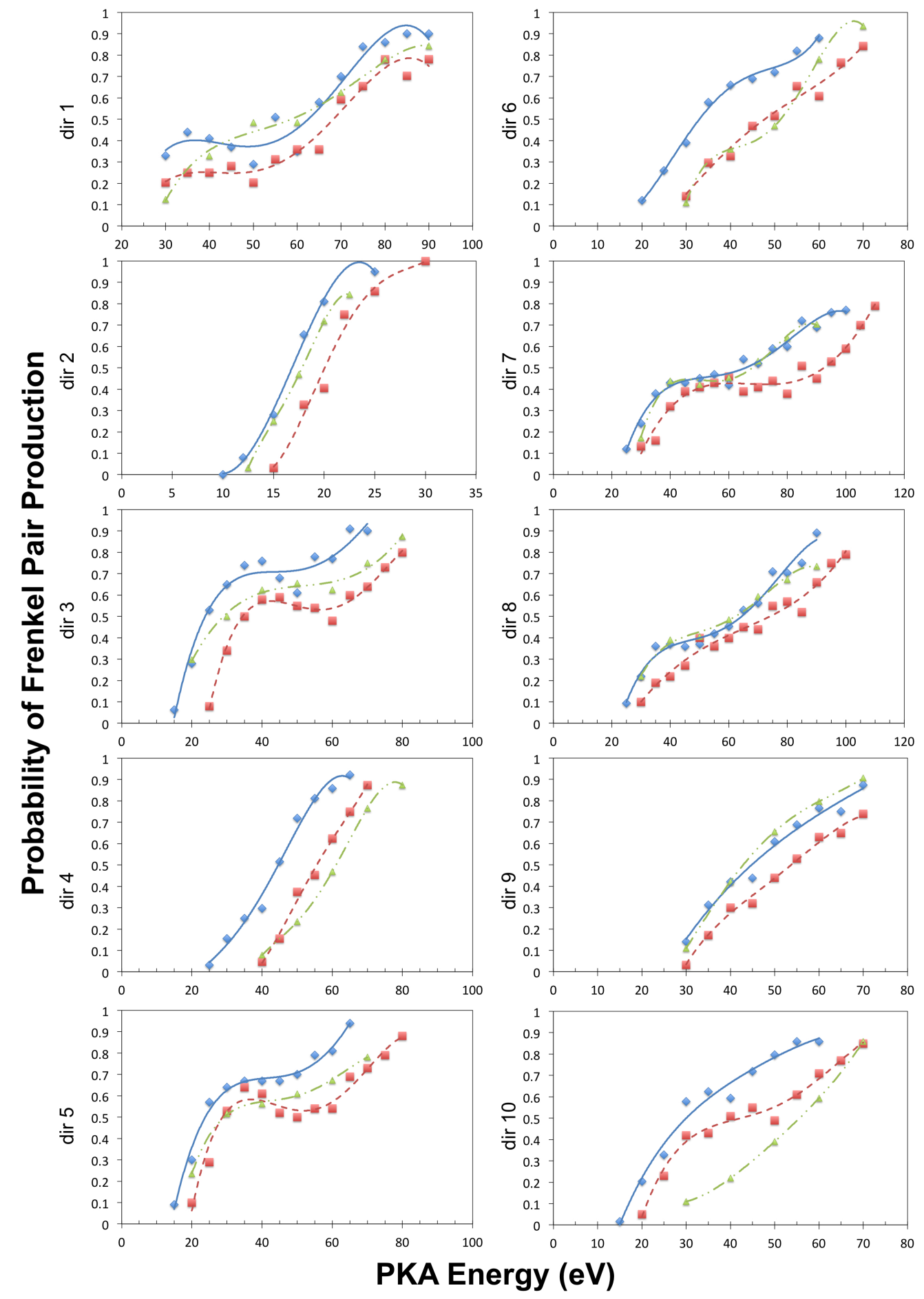

Figure 1: Probability of Frenkel pair production as a function of PKA energy at $300 \mathrm{~K}$ for ten random PKA directions. The directions are labeled from dir 1 to $\operatorname{dir} 10$. The red square data points and the red dashed line denote a system without external applied strain. The blue diamond data points and the blue solid line denote a system with $2 \%$ hydrostatic expansion. The green triangle data points and green dashed line denote a system with $5 \%$ Bain strain. Trend lines are fourth-order polynomials. 
This highlights the strong crystallographic dependence of the TDE and the need for investigation over a variety of PKA directions.

Using the data in Figure 1, we want extract a value for the TDE for each direction. The point at which the probability curve becomes greater than or equal to 0.5 is taken as the value of the TDE for that specific PKA direction. Thus, the TDE is the energy when it becomes probable that a defect is created. This data is tabulated in Table 1 for systems at $300 \mathrm{~K}$ and in Table 2 for systems at $500 \mathrm{~K}$.

Table 1: The threshold displacement energy as a function of direction at $300 \mathrm{~K}$ for three strain conditions. Energies given in $\mathrm{eV}$.

\begin{tabular}{|c|c|c|c|}
\hline & Unstrained & Hydrostatic 2\% & Bain 5\% \\
\hline dir 1 & 68 & 63 & 59 \\
$\operatorname{dir} 2$ & 20 & 17 & 18 \\
$\operatorname{dir} 3$ & 29 & 24 & 29 \\
$\operatorname{dir} 4$ & 56 & 45 & 61 \\
$\operatorname{dir} 5$ & 29 & 24 & 29 \\
$\operatorname{dir} 6$ & 48 & 33 & 51 \\
$\operatorname{dir} 7$ & 93 & 64 & 68 \\
$\operatorname{dir} 8$ & 74 & 64 & 62 \\
$\operatorname{dir} 9$ & 54 & 46 & 46 \\
$\operatorname{dir} 10$ & 42 & 30 & 56 \\
\hline
\end{tabular}


Table 2: The threshold displacement energy as a function of direction at $500 \mathrm{~K}$ for three strain conditions. Energies given in $\mathrm{eV}$.

\begin{tabular}{|c|c|c|c|}
\hline & Unstrained & Hydrostatic 2\% & Bain 5\% \\
\hline dir 1 & 81 & 54 & 78 \\
$\operatorname{dir} 2$ & 23 & 18 & 22 \\
$\operatorname{dir} 3$ & 68 & 30 & 60 \\
$\operatorname{dir} 4$ & 61 & 52 & 68 \\
$\operatorname{dir} 5$ & 65 & 49 & 39 \\
$\operatorname{dir} 6$ & 58 & 46 & 53 \\
$\operatorname{dir} 7$ & 89 & 77 & 67 \\
$\operatorname{dir} 8$ & 82 & 69 & 57 \\
$\operatorname{dir} 9$ & 60 & 51 & 42 \\
$\operatorname{dir} 10$ & 54 & 37 & 59 \\
\hline
\end{tabular}

The probability curves displayed in Figure 1 are arithmetically averaged, creating a single angle-integrated probability curve for each of the three strain conditions. The resulting three probability curves are displayed in Figure 2, for the system at $300 \mathrm{~K}$ and $500 \mathrm{~K}$. In order to determine the value of $\mathrm{E}_{\mathrm{d} \text {,med }}^{\mathrm{pp}}$, a forrth order polynomial is fit to the data and the $\mathrm{E}_{\mathrm{d} \text {,med }}^{\mathrm{pp}}$ is calculated from the point where the polynomial fit crosses 0.5 probability.
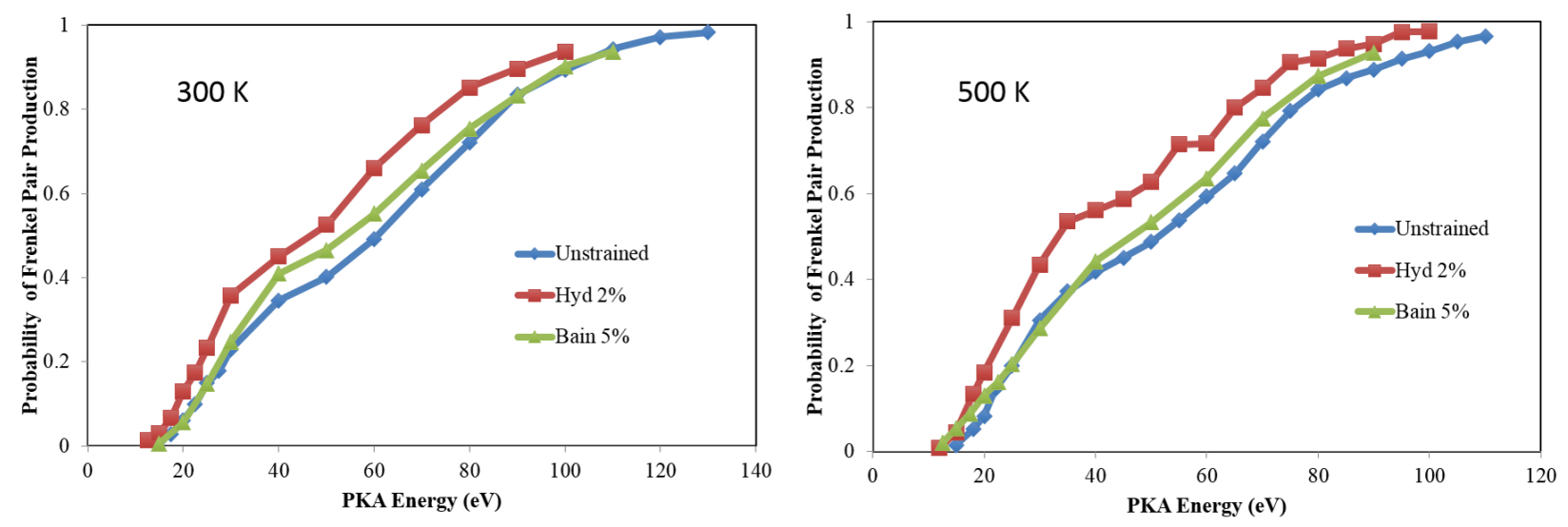

Figure 2: The angle-integrated probability curves at $300 \mathrm{~K}$ and $500 \mathrm{~K}$ for three strain conditions. The blue diamond data points denote a system without external applied strain. The red square data points denote a system with $2 \%$ hydrostatic expansion. The green triangle data points denote a system with $5 \%$ Bain strain.

Figure 2 shows that with applied hydrostatic expansion, there is a marked shift up of the probability curve for both temperatures. Thus, for a given PKA energy, it is more likely to create a Frenkel pair in a system under $2 \%$ hydrostatic expansion, than in an unstrained system. This is consistent with previous 
work [21. This also illustrates that there is an overall decrease of approximately $14 \mathrm{eV}$ in the TDE at 300 $\mathrm{K}$ and a decrease of approximately $13 \mathrm{eV}$ at $500 \mathrm{~K}$ that would be used as an input into the Kinchin-Pease or NRT radiation damage models. For applied Bain strain at $300 \mathrm{~K}$, there exists a very minor shift up of the probability curve, yielding minimal changes to the average radiation damage response of the material. This is also consistent with previous work 21. However, the shift is more substantial for simulations at 500 K. Application of $5 \%$ Bain strain at $500 \mathrm{~K}$ yields a decrease in the $\mathrm{E}_{\mathrm{d} \text {,med }}^{\mathrm{pp}}$ of $5 \mathrm{eV}$.

The results for temperatures at $300 \mathrm{~K}$ and $500 \mathrm{~K}$ from Figure 2 are tabulated in Table 3 . The values of $\mathrm{E}_{\mathrm{d} \text {,med }}^{\mathrm{pp}}$ at $300 \mathrm{~K}$ for the unstrained, hydrostatic $2 \%$ and Bain $5 \%$ are $50.0 \mathrm{eV}, 35.8 \mathrm{eV}$ and $46.2 \mathrm{eV}$, respectively. The values of $\mathrm{E}_{\mathrm{d} \text {,med }}^{\mathrm{pp}}$ at $500 \mathrm{~K}$ for the unstrained, hydrostatic $2 \%$ and Bain $5 \%$ are $57.4 \mathrm{eV}$, $44.4 \mathrm{eV}$ and $51.7 \mathrm{eV}$, respectively. The standard value for the TDE in iron is usually taken as $40 \mathrm{eV}$ [42, 43]. This value is for a system at $0 \mathrm{~K}$, and thus thermal fluctuations at $300 \mathrm{~K}$ and $500 \mathrm{~K}$ are dictating an increase in the TDE to a value of $50 \mathrm{eV}$ and $57 \mathrm{eV}$, respectively. Nordlund [26] calculated the $\mathrm{E}_{\mathrm{d} \text {,med }}^{\mathrm{pp}}$ at $36 \mathrm{~K}$ in an unstrained system utilizing the Ackland $\mathrm{ABC}$ potential [27, resulting in a value of $44 \mathrm{eV}$. This corresponds to our results in that an increase in temperature is yielding an increase in the TDE. It should be noted that the effect of temperature on the TDE is much larger than the thermal energy.

For all strain conditions, an increase in temperature yields an increase in the $\mathrm{E}_{\mathrm{d}, \mathrm{ped}}^{\mathrm{p}}$. For both temperatures, the unstrained system exhibits the highest displacement energy and the system under $2 \%$ hydrostatic expansion exhibits the lowest displacement energy. The largest variance in the TDE with respect to strain has a magnitude of $14.2 \mathrm{eV}$. The largest variance in the TDE with respect to temperature is $8.6 \mathrm{eV}$. In the Kinchin-Pease and NRT equations, the number of displacements is inversely proportional to the TDE. If we take as an example the unstrained system of BCC Fe, using the TDE value at $300 \mathrm{~K}$ yields approximately $18 \%$ more displacements than if the value of TDE at $500 \mathrm{~K}$ is utilized, and $18 \%$ fewer displacements than if the typical value for $\mathrm{BCC} F$ of $40 \mathrm{eV}$ is utilized. This underlines the importance of the proper implementation of the appropriate $\mathrm{E}_{\mathrm{d} \text {,med }}^{\mathrm{pp}}$ for a particular system.

In order to determine the statistical significance of these results, the standard error of the mean was determined for each data point in Figure 2. The standard error of the mean for each data point was added to that respective data point in order to create a higher bound probability curve. Likewise a lower bound probability curve was created by subtracting the standard error of the mean for each respective data point. The upper bound and lower bound $\mathrm{E}_{\mathrm{d}, \text { med }}^{\mathrm{pp}}$ is calculated from the point where the upper bound and lower bound probability curves, respectively, cross 0.5 probability. This can provide us with a most probable range for the values of $\mathrm{E}_{\mathrm{d} \text {,med }}^{\mathrm{pp}}$. These ranges are tabulated in Table 3. This data shows that although there is a slight depression in the TDE due to applied 5\% Bain strain, this decrease is not statistically significant given the finite set of PKA directions analyzed. However, this data confirms that the decrease in the TDE due to applied $2 \%$ hydrostatic expansion is statistically significant. 
Table 3: The median threshold displacement energy in BCC Fe at three strain conditions and two temperatures. Energies given in $\mathrm{eV}$. The range of \pm one standard deviation is given in parentheses.

\begin{tabular}{|c|c|c|}
\hline & $300 \mathrm{~K}$ & $500 \mathrm{~K}$ \\
\hline Unstrained & $50.0(45.1-54.7)$ & $57.4(45.1-54.7)$ \\
Hyd 2\% & $35.8(30.2-40.9)$ & $44.4(38.2-50.0)$ \\
Bain 5\% & $46.2(38.8-52.3)$ & $51.7(44.0-59.3)$ \\
\hline
\end{tabular}

\subsection{Lower Bound of the Threshold Displacement Energy}

Typically, the direct experimental measurements of the TDE measure the lowest incident electron energy where a defect signal can be detected, for example by changes in resistivity. This is analogous to the lower bound of the threshold displacement energy, $E_{d}^{1}$. The $E_{d}^{l}$ is the lowest possible value of kinetic energy such that a Frenkel pair can be created. In this work, the minimum probability to meet the criterion for defect production is 0.01 . The results for the $\mathrm{E}_{\mathrm{d}}^{\mathrm{l}}$ as a function of PKA direction and temperature are displayed in Figure 3, for all three strain conditions.

From Figure 3, it is found that the magnitude of $\mathrm{E}_{\mathrm{d}}^{\mathrm{l}}$ varies from $10 \mathrm{eV}$ at the minimum to $26 \mathrm{eV}$ at the maximum across all PKA directions and temperatures. There is a strong dependence of the $\mathrm{E}_{\mathrm{d}}^{1}$ on crystallographic direction. These results are numerically averaged to determine the average $\mathrm{E}_{\mathrm{d}}^{\mathrm{l}}$ for each strain condition and temperature. These results are tabulated in Table 4.
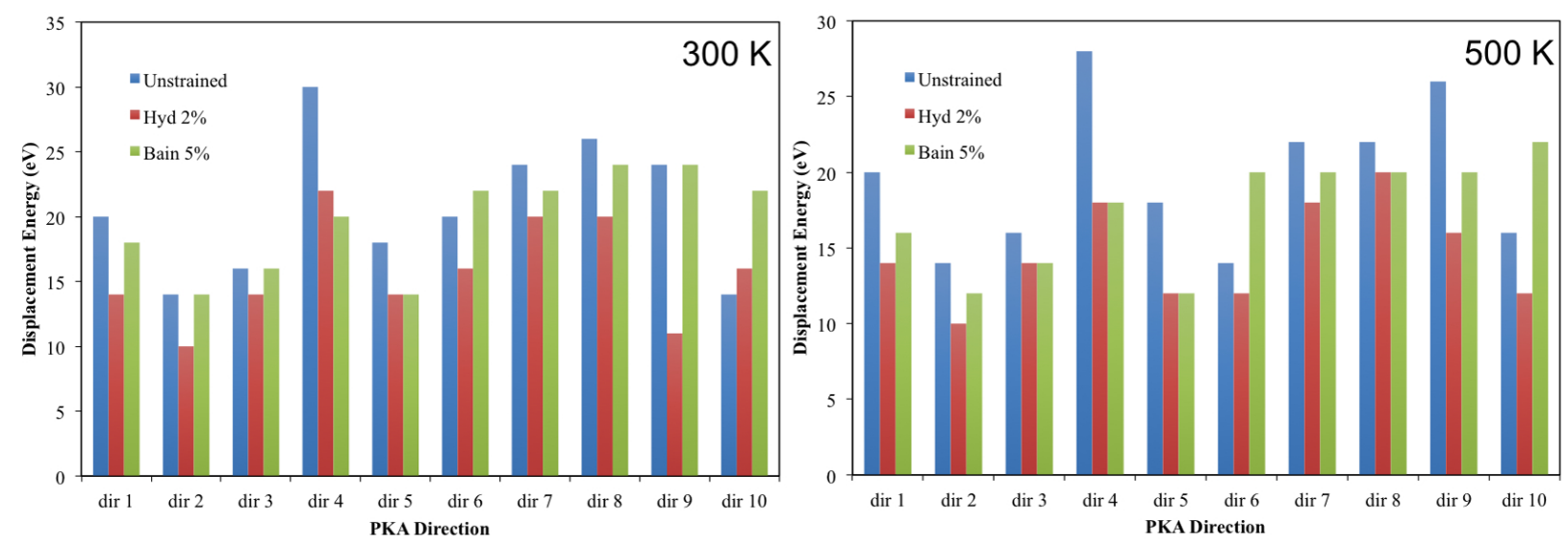

Figure 3: The lower bound threshold displacement energy as a function of direction at $300 \mathrm{~K}$ and $500 \mathrm{~K}$. The blue bars denote a system without external applied strain. The red bars denote a system with $2 \%$ hydrostatic expansion. The green bars denote a system with $5 \%$ Bain strain.

For both temperatures, the unstrained system exhibits the highest displacement energy and the system under $2 \%$ hydrostatic expansion exhibits the lowest displacement energy, consistent with the results in Table 3 for $\mathrm{E}_{\mathrm{d}, \text { med }}^{\mathrm{pp}}$. However, for all strain conditions, the $\mathrm{E}_{\mathrm{d}}^{\mathrm{l}}$ at $300 \mathrm{~K}$ is higher than at $500 \mathrm{~K}$. This is in direct contrast to the results in Table 3. Thus, the $\mathrm{E}_{\mathrm{d}}^{\mathrm{l}}$ and the $\mathrm{E}_{\mathrm{d} \text {,med }}^{\mathrm{pp}}$ exhibit opposite behaviors as a function of 
Table 4: The lower bound of the threshold displacement energy in BCC Fe at three strain conditions and two temperatures. Energies given in eV. Plus/minus is the standard error of the mean.

\begin{tabular}{|c|c|c|}
\hline & $300 \mathrm{~K}$ & $500 \mathrm{~K}$ \\
\hline Unstrained & $20.6 \pm 1.69$ & $19.6 \pm 1.54$ \\
Hyd $2 \%$ & $15.7 \pm 1.25$ & $14.6 \pm 1.03$ \\
Bain 5\% & $19.6 \pm 1.22$ & $17.4 \pm 1.16$ \\
\hline
\end{tabular}

temperature from $300 \mathrm{~K}$ to $500 \mathrm{~K}$. This can be explained by the nature of these two different definitions of the TDE. For $\mathrm{E}_{\mathrm{d} \text {,med }}^{\mathrm{pp}}$, higher energy PKAs are utilized, inducing mini-cascades. Additional thermal fluctuations can allow for increased recombination as the thermal spike anneals. For $\mathrm{E}_{\mathrm{d}}^{1}$, increased thermal fluctuations can act to rapidly diffuse a created interstitial away from the resultant vacancy, creating a stable Frenkel pair. Thus, an increase in temperature yields an increase in the probability for a set of PKAs to create a single Frenkel pair $\left(\mathrm{E}_{\mathrm{d}}^{\mathrm{l}}\right)$, while yielding a decrease in the overall probability of creating a Frenkel pair $\left(\mathrm{E}_{\mathrm{d}, \mathrm{ped}}^{\mathrm{pp}}\right)$.

The traditional value used for the $\mathrm{E}_{\mathrm{d}}^{\mathrm{l}}$ in $\mathrm{BCC} \mathrm{Fe}$ is $20 \mathrm{eV}$ [42]. This work shows that the absolute minimum is $14 \mathrm{eV}$ for an unstrained system, which is a significantly lower value than the traditional $\mathrm{E}_{\mathrm{d}}^{\mathrm{l}}$. However, the averaged results at $300 \mathrm{~K}$ and $500 \mathrm{~K}$ for the unstrained system are in line with this traditional value. The maximum variance in the $\mathrm{E}_{\mathrm{d}}^{\mathrm{l}}$ as a function of strain is $5 \mathrm{eV}$. The maximum variance in the $\mathrm{E}_{\mathrm{d}}^{1}$ as a function of temperature is $2 \mathrm{eV}$.

\section{Conclusions}

In this study, molecular dynamics simulations were performed on pure BCC Fe to investigate the effects of applied strain and temperature on the threshold displacement energy. Two separate values for the threshold displacement energy are calculated: the median value of the displacement energy $\left(\mathrm{E}_{\mathrm{d}, \mathrm{ped}}^{\mathrm{pp}}\right)$ and the lower bound of the displacement energy $\left(E_{d}^{1}\right)$. Each threshold displacement energy is determined as a function of ten PKA directions, three strain conditions, and two temperatures. It was determined that for $\mathrm{E}_{\mathrm{d} \text {,med }}^{\mathrm{pp}}$, application of $2 \%$ hydrostatic strain results in a decrease of up to $14 \mathrm{eV}$ and application of $5 \%$ Bain strain results in a decrease of up to $6 \mathrm{eV}$. For $\mathrm{E}_{\mathrm{d}, \text { med }}^{\mathrm{pp}}$, an increase in the temperature of the system from $300 \mathrm{~K}$ to $500 \mathrm{~K}$ can result in an increase of up to $9 \mathrm{eV}$. For $\mathrm{E}_{\mathrm{d}}^{\mathrm{l}}$, application of $2 \%$ hydrostatic strain results in a decrease of up to $5 \mathrm{eV}$ and application of $5 \%$ Bain strain results in a decrease of up to $2 \mathrm{eV}$. For $\mathrm{E}_{\mathrm{d}}^{\mathrm{l}}$, an increase in the temperature of the system from $300 \mathrm{~K}$ to $500 \mathrm{~K}$ can result in a decrease of up to $2 \mathrm{eV}$. This study clearly shows the importance of accounting for changes in strain environment and system temperature regarding the threshold displacement energy and the resulting radiation damage behavior. 


\section{Acknowledgement}

This work was supported by the US Department of Energy, project \#DE-NE0000536000.

\section{Appendix A. Set of random directions}

The possible set of PKA directions in the body-centered cubic crystal structure can be defined by two angles, $\theta$ and $\phi$, as illustrated in Figure A.4. The set of PKA directions is chosen by utilizing a random number generator to select $\theta$ and $\phi$ values, varying from 0 to $\pi / 2$ and 0 to $\pi / 4$, respectively. The specific $\theta$ and $\phi$ values are provided in Table A.5, along with the corresponding $h, k, l$ values. These $h, k, l$ values are normalized such that $h$ is equal to 1 . It was found that this set of directions adequately samples the BCC crystal structure. Tests were performed utilizing a larger subset of directions, yielding negligible variance in results.

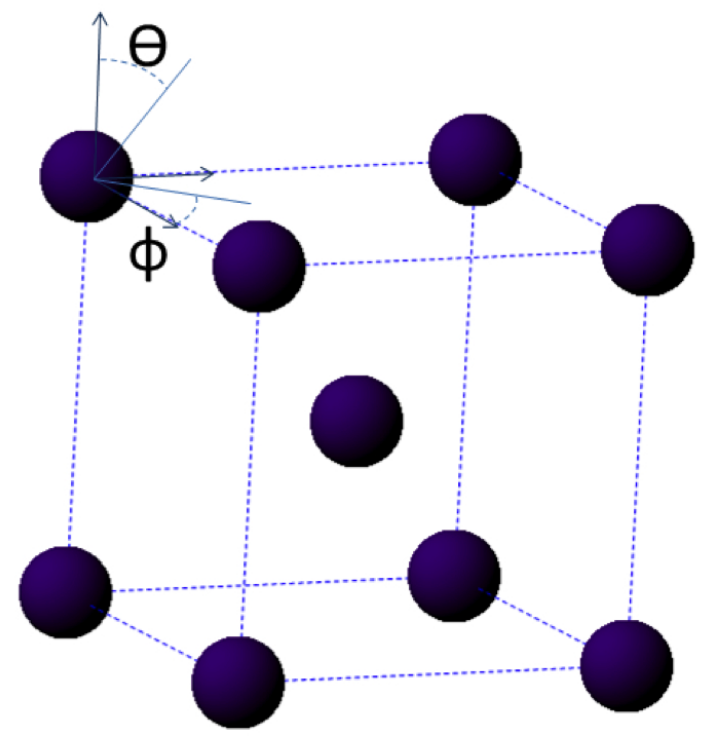

Figure A.4: Random directions were selected for primary knock-on atoms to sample the complete set of directions within a BCC crystal system. The angle theta $(\theta)$ was allowed to vary from 0 to $\pi / 2$. The angle phi $(\phi)$ was allowed to vary from 0 to $\pi / 4$. 
Table A.5: The set of ten random directions utilized in all simulations. Values of $\theta$ and $\phi$ are given in degrees. Values of $h, k, l$ are normalized such that $h$ is equal to 1 .

\begin{tabular}{|c|c|c|c|c|c|}
\hline & $\theta$ & $\phi$ & $\mathrm{h}$ & $\mathrm{k}$ & $\mathrm{l}$ \\
\hline $\operatorname{dir} 1$ & 27 & 24 & 1 & 2.25 & 4.83 \\
$\operatorname{dir} 2$ & 17 & 5 & 1 & 11.43 & 37.53 \\
$\operatorname{dir} 3$ & 24 & 15 & 1 & 3.73 & 8.68 \\
$\operatorname{dir} 4$ & 31 & 11 & 1 & 5.14 & 8.72 \\
$\operatorname{dir} 5$ & 24 & 14 & 1 & 4.01 & 9.28 \\
$\operatorname{dir} 6$ & 86 & 26 & 1 & 2.05 & 0.16 \\
$\operatorname{dir} 7$ & 37 & 38 & 1 & 1.28 & 2.16 \\
$\operatorname{dir} 8$ & 35 & 44 & 1 & 1.04 & 2.06 \\
$\operatorname{dir} 9$ & 85 & 45 & 1 & 1 & 0.12 \\
$\operatorname{dir} 10$ & 65 & 4 & 1 & 14.30 & 6.68 \\
\hline
\end{tabular}

[1] D. Bacon, A. Calder, J. Harder, S. Wooding, Computer simulation of low-energy displacement events in pure bcc and hcp metals, J. Nucl. Mater. 205 (1993) 52-58.

[2] R. Stoller, Point defect survival and clustering fractions obtained from molecular dynamics simulations of high energy cascades, J. Nucl. Mater. 233 (1996) 999-1003.

[3] F. Gao, D. Bacon, A. Calder, P. Flewitt, T. Lewis, Computer simulation study of cascade overlap effects in alpha-iron, J. Nucl. Mater. 230 (1996) 47-56.

[4] F. Gao, D. Bacon, P. Flewitt, T. Lewis, A molecular dynamics study of temperature effects on defect production by displacement cascades in alpha-iron, J. Nucl. Mater. 249 (1997) 77-86.

[5] C. Becquart, K. Decker, C. Domain, J. Ruste, Y. Souffez, J. Turbatte, J. V. Duysen, Massively parallel molecular dynamics simulations with eam potentials, Rad. Eff. Def. Sol. 142 (1997) 9-21.

[6] R. Stoller, L. Greenwood, Subcascade formation in displacement cascade simulations: Implications for fusion reactor materials, J. Nucl. Mater. 271 (1999) 57-62.

[7] L. Malerba, Molecular dynamics simulation of displacement cascades in alpha-fe: A critical review, J. Nucl. Mater. 351 (2006) 28-38.

[8] R. Averback, T. de la Rubia, Displacement damage in irradiated metals and semiconductors, Solid State Physics 51 (1998) 281.

[9] W. Phythian, R. Stoller, A. Foreman, A. Calder, D. Bacon, A comparison of displacement cascades in 
copper and iron by molecular-dynamics and its application to microstructural evolution, J. Nucl. Mater. 223 (1995) 245.

[10] D. Bacon, T. de la Rubia, Molecular-dynamics computer-simulations of displacement cascades in metals, J. Nucl. Mater. 216 (1994) 275.

[11] M. Caturla, N. Soneda, E. Alonso, B. Wirth, T. de la Rubia, Comparative study of radiation damage accumulation in cu and fe, J. Nucl. Mater. 276 (2000) 13.

[12] S. Zinkle, B. Singh, Analysis of displacement damage and defect production under cascade damage conditions, J. Nucl. Mater. 199 (1993) 173.

[13] E. Zarkadoula, S. Daraszewicz, D. Duffy, M. Seaton, I. Todorov, K. N. M. Dove, K. Trachenko, The nature of high-energy radiation damage in iron, J. Phys.: Cond. Mat. 25 (2013) 125402.

[14] N. Soneda, T. de la Rubia, Defect production, annealing kinetics and damage evolution in alpha-fe: an atomic-scale computer simulation, Phil. Mag. A 78 (1998) 995.

[15] D. Bacon, Y. Osetsky, Multiscale modelling of radiation damage in metals: from defect generation to material properties, Mat. Sci. Eng. A 365 (2004) 46.

[16] M. Robinson, N. Marks, G. Lumpkin, Sensitivity of the threshold displacement energy to temperature and time, Phys. Rev. B (2012) 134105.

[17] E. Hayward, C. Deo, A molecular dynamics study of irradiation induced cascades in iron containing hydrogen, Comp., Mater. and Cont. 16 (2010) 101-115.

[18] G. Ackland, M. Mendelev, D. Srolovitz, S. Han, A. Barashev, Development of an interatomic potential for phosphorus impurities in -iron, J. Phys.: Cond. Mat. 16 (2004) S2629.

[19] D. Terentyev, L. Malerba, R. Chakarova, Displacement cascades in fe-cr: A molecular dynamics study, J. Nucl. Mater. 349 (2006) 119.

[20] Y. Yang, X. Tang, F. Chen, H. Huang, J. Liu, A molecular dynamics-based comparison of defect production in collision cascades during the bombardment of iron with different ions, Nucl. Sci. and Tech. 57 (29).

[21] B. Beeler, M. Asta, P. Hosemann, N. Gronbech-Jensen, Effects of applied strain on radiation damage generation in body-centered cubic iron, J. Nucl. Mater. 459 (2015) 159.

[22] S. Miyashiro, S. Fujita, T. Okita, Md simulations to evaluate the influence of applied normal stress or deformation on defect production rate and size distribution of clusters in cascade process for pure cu, J. Nucl. Mater. 415 (2011) 1-4. 
[23] S. Di, Z. Yao, M. Daymond, F. Gao, Molecular dynamics simulations of irradiation cascades in alphazirconium under macroscopic strain, Nucl. Instr. Meth. B 303 (2013) 95-99.

[38] J. Ziegler, J. Biersack, U. Littmark, Stopping and Ranges of Ions in Matter, Pergamon Press, 1985.

[25] M. Norgett, M. Robinson, L. Torrens, A proposed method of calculating displacement dose rates, Nucl. Eng. Design 33 (1975) 50-54.

[26] K. Nordlund, J. Wallenius, L. Malerba, Molecular dynamics simulation of threshold displacement energies in fe, Nucl. Instr. Meth. B 246 (2006) 322-332.

[27] G. Ackland, D. Bacon, A. Calder, T. Harry, Computer simulation of point defect properties in dilute fe-cu alloy using a many-body interatomic potential, Phil. Mag. 75 (1997) 713.

[28] K. Zolnikov, A. Korchuganov, D. Kryzhevich, V. Chernov, S. Psakhie, Structural change in elastically stressed crystallites under irradiation, Nucl. Instr. Meth. B doi:10.1016/j.nimb.2015.01.029.

[29] B. Liu, F. Yuan, K. Jin, Y. Zhang, W. Weber, Ab initio molecular dynamics investigations of low-energy recoil events in ni and nico, J. Phys.: Cond. Mat. 27 (2015) 435006.

[30] D. Delgado, R. Vila, Statistical molecular dynamics study of displacement energies in diamond, J. Nucl. Mater. 419 (2011) 32.

[31] M. Robinson, N. Marks, K. Whittle, G. Lumpkin, Systematic calculation of threshold displacement energies: case study in rutile, Phys. Rev. B 85 (2012) 104105.

[32] Z. Chen, N. Kioussis, N. Ghoniem, D. Seif, Strain-field effects on the formation and migration energies of self interstitials in alpha fe from first principles, Phys. Rev. B 81 (2010) 094102.

[33] F. Abraham, Computational statistical mechanics: Methodology, apllications and supercomputing, Adv. in Phys. 35 (1986) 1.

[34] M. Allen, D. Tildesley, Computer Simulation of Liquids, Clarendon Press, Oxford, 1987.

[35] S. Plimpton, Fast parallel algorithms for short-range molecular dynamics, J. Comp. Phys. 117 (1995) $1-19$.

[36] M. Daw, M. Baskes, Embedded-atom method - derivation and application to impurities, surfaces, and other defects in metals, Phys. Rev. B 29 (1984) 6443.

[37] M. Mendelev, S. Han, D. Srolovitz, G. Ackland, D. Sun, M. Asta, Development of new interatomic potentials appropriate for crystalline and liquid iron, Phil. Mag. 83 (2003) 3977-3994. 
[39] N. Gronbech-Jensen, O. Farago, A simple and effective verlet-type algorithm for simulating langevin dynamics, Mol. Phys. 111 (2013) 983.

[40] N. Gronbech-Jensen, N. Hayre, O. Farago, Application of the g-jf discrete-time thermostat for fast and accurate molecular simulations, Comp. Phys. Comm. 185 (2014) 524.

[41] L. Malerba, J. Perlado, Basic mechanisms of atomic displacement production in cubic silicon carbide: A molecular dynamics study, Phys. Rev. B 65 (2002) 045202.

[42] G. Was, Fundamentals of Radiation Materials Science, Springer, 2007.

[43] Astm standard e693-94, standard practice for characterising neutron exposure in iron and low alloy steels in termins of displacements per atom (dpa), 1994. 